\title{
Search for UHE Photons with the Telescope Array Hybrid Detector
}

\author{
Katsuya Yamazaki* for the Telescope Array collaboration ${ }^{\dagger}$ \\ Earthquake Research Institute, The University of Tokyo, Tokyo, Japan \\ E-mail: kyamazaki@eri.u-tokvo.ac.ip
}

\begin{abstract}
The origin of ultra-high energy cosmic rays are still unknown, and this is one of the biggest problem in the area of ultra-high energy cosmic ray research field. One of the reasons is magnetic deflection, because most of the cosmic rays are charged particles and the universe is filled with galactic and extragalactic magnetic fields. On the other hand, there is no magnetic deflection to the photons, so that their trajectories can be traced back to their origins. Therefore, we search for ultra-high energy photons with the Telescope Array experiment in order to understand origin of ultra-high energy cosmic rays. The Telescope Array is a hybrid detector consisting of an array of scintillation detectors, which measure the lateral profile of air showers, and fluorescence detectors, which measure the longitudinal profile of air showers. We use a parameter called $X_{\max }$, which is the depth of maximal point of shower development, to search for photon-like events. We will report on the analysis method, and the result of a photon search using five years of TA data.
\end{abstract}

The 34th International Cosmic Ray Conference,

30 July- 6 August, 2015

The Hague, The Netherlands

\footnotetext{
*Speaker.

${ }^{\dagger}$ For full author list see http://www.telescopearray.org/images/papers/ICRC2015-authorlist.pdf
} 


\section{Introduction}

The origin of ultra-high energy (UHE) cosmic rays has been sought for many years, and it is one of the most important topics in the UHE cosmic ray research field. Recently, the Telescope Array (TA) experiment has published a result consistent with the existence of anisotropy in the arrival directions of UHE cosmic rays However, sources of UHE cosmic rays are still unknown. One of the reasons is magnetic deflection in their trajectories. Since, the universe is filled with galactic and extragalactic magnetic fields, charged particles are deflected by these magnetic fields even though they are UHE cosmic rays. On the other hand, photons are not deflected by magnetic fields, and their trajectories can be traced back to their origins.

UHE photons have not been discovered yet, however, UHE photon emission is predicted by some UHE cosmic ray source models. There are basically two types of models. One is the nonacceleration model called "top-down" scenario. For instance, decay/annihilation of the relic Topological Defects (TD)[园] and Super Heavy Dark Matter (SHDM)[3], or resulting from resonant production of $\mathrm{Z}$ boson (Z-Burst, ZB) [䧃]. In contrast, the other type of model involves acceleration and is known as "bottom-up" scenario. In this scenario, UHE photons is produced by the GZK process from $\pi^{0}$ decays. These scenarios can be distinguished by the different flux of UHE photons. The UHE photon fluxes expected for the top-down models are significantly larger than that for bottom-up scenario. Also, within these scenarios we can examine the ratio of UHE photons to other UHE cosmic rays.

In this work, we search for UHE photons using the air shower profile observed by Fluorescence Detectors (FDs) in the Telescope Array (TA) experiment.

\section{Telescope Array Experiment}

The Telescope Array (TA) experiment is the largest observatory for UHE cosmic rays in the northern hemisphere, and it is located at $39.3^{\circ} \mathrm{N}, 112.9^{\circ} \mathrm{W}$, and about $1400 \mathrm{~m}$ above sea level, Utah, USA. The experiment is operated by the international collaboration of Japan, USA, Korea, Russia, and Belgium, since 2008. TA consists of three fluorescence detector (FD) stations[[]] and an array of 507 surface detectors (SDs)[G]. The area covered by the SD array is about $700 \mathrm{~km}^{2}$, and the FDs are located at around the SD array viewing over the SD array. The FDs and SDs observe cosmic ray air showers, and each sampled data are used complementary to reconstruct the air showers with hybrid analysis method.

\section{Monte Carlo Simulation for Hybrid Event}

There are three steps for the hybrid event simulation in this work: simulation of extensive air showers (EASs), calculation of detector responses for SDs, and detector response simulation for FDs.

EASs are simulated with CORSIKA package[प]. For primary cosmic rays of energies above $10^{18} \mathrm{eV}$, the number of secondary particles becomes too large to trace all of the particles. Therefore, we use the thinning option to reduce the number of secondary particles to trace. The thinning factor of $10^{-6}$ is used for CORSIKA simulation in this work. The hadronic interaction model used in this work is QGSJETII-O3[8]]. 
The thinning causes non-trivial fluctuation to the lateral distribution of secondly particles. In order to correct this situation, a method has been developed by the TA collaboration to recover the information lost by thinning and to successfully use thinned showers for practical simulations, and this process is called dethinning[Q] . Dethinned CORSIKA simulations are used for the SD response simulations using GEANT4. Each CORSIKA shower is used repeatedly with random different core positions to reduce the calculation time. For SD simulations with proton primaries, each proton shower is also used repeatedly with random different azimuthal angle. On the other hand, for photon primaries, the CORSIKA showers are not used repeatedly with azimuthal angle, because shower development depends on their azimuthal angles because of preshower effect[ए]], which causes electron-positron pair production by interacting with geomagnetic field at above the atmosphere.

The longitudinal development of EASs simulated with CORSIKA is used in the FD simulations using a software developed by the TA collaboration. Energy depositions are converted to fluorescence photons taking into account the wavelength spectrum, and parameterized atmospheric conditions. These photons are traced to the PMTs on FDs and simulated signals are produced.

\section{Hybrid Event Reconstruction}

The hybrid event reconstruction consists of four steps[]]: PMT selection, shower geometry reconstruction, longitudinal shower profile reconstruction, and quality cuts. The timing information of one triggered SD and time profile obtained by FDs are used to reconstruct the shower geometry. It allows significant improvement in the accuracy of the determination of shower geometries compared with the FD monocular analysis.

After the geometrical reconstruction, the longitudinal profile of the EAS is reconstructed using the FD data. In order to reconstruct longitudinal profile, reconstructed geometry and FD PMT signal profile are used in the Inverse MC (IMC) technique[[2]. This IMC technique iteratively searches for the optimum parameters of longitudinal profile, which best matches the observed shower image.

There are inaccurately reconstructed events even though reconstruction process is successfully finished. For example, for an event of which true $X_{\max }$ is outside of the field of view (FOV) of FDs, it is difficult to estimate $X_{\max }$ with accuracy. Therefore, we discard these inaccurately reconstructed events from data set in order to avoid using these inaccurate events for further analyses. Additional quality cuts for the event selection are as follows:

- The number of selected PMTs $>20$

- The estimated $X_{\max }$ is inside of the FOV of the FD

- The estimated incident zenith angle $<55$ degrees

- The estimated core position is inside of the SD array

- The minimum viewing angle (see text) $>20$ degrees

The minimum viewing angle is the minimum angle between the shower axis and the viewing angle from the FD to the each point of shower axis within the inside of the FOV. This cut reduces events which have photon component dominated by the Cherenkov photons, because for such events, because it is difficult to reconstruct the longitudinal profile of fluorescence photons with accuracy for such events. 


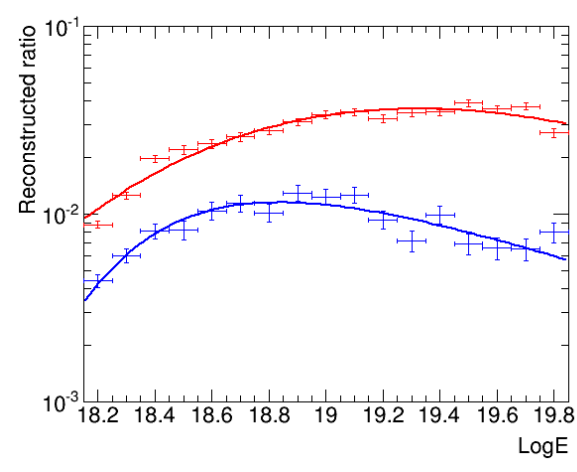

Figure 1: The survival fraction $R$. Red and blue points indicate $R$ for protons and that for photons, respectively. The lines show the fit results with an empirical equation.

\section{Photon-like Event Selection}

Our quality cut requires the reconstructed $X_{\max }$ to be inside the FOV of FDs, thus deeplypenetrating EASs are discarded by this criterion because the shower maximum position may be lower than the FOV. Since most of EASs of primary photons have significantly deeper $X_{\max }$ than that of primary protons at energies greater than $1 \mathrm{EeV}$, photon primary EASs are more likely to be discarded with this criterion. Therefore, we applied an additional cut criterion discarding the events which have smaller reconstructed zenith angle than $20^{\circ}$ (photon enhancement cut), to suppress acceptance bias between protons and photons. The acceptance bias is calculated comparing the survival fraction, $R$, for photon primaries to that for proton primaries. The fraction $R$ is defined as the ratio of the number of remaining events after the reconstructions and quality cuts to the number of thrown MC events, i.e., $R=N_{\text {reconstructed }} / N_{\text {thrown }}$. Figure $\mathbb{U}$ shows $R$ for proton and photon primaries. As a result, we get the acceptance bias as the ratio of $R$ for photons to that for protons (QGSJETII-03) as 0.312, 0.302, 0.280, and 0.248 at energies grater than 2, 3, 5, and 10 $\mathrm{EeV}$, respectively.

After the photon enhancement cut, a photon-like event selection criterion is determined by MC simulation for photon primaries. We calculated the averages of reconstructed $X_{\max }$ for photon MC, and adopted it to the photon selection criterion. Figure $\square$ (left) shows the averaged $X_{\max }$ of the photon primary EASs. The fitting result of the averaged $X_{\max }$ of each energy bin for the photon primaries is the photon selection criterion in this work. When the reconstructed $X_{\max }$ of an EAS is larger than the selection criterion at the energy range of the event, the event is tagged as photon-like event. Selection efficiencies for primary photons are $0.476,0.477,0.500$, and 0.479 at energies greater than 2, 3, 5, and $10 \mathrm{EeV}$, respectively. In Figure $\square$ (right), the selection criterion and reconstructed $X_{\max }$ of proton primary EASs are plotted. In order to estimate the proton contamination with this photon selection criterion, the photon selection is applied to the proton MC (QGSJETII-03) data set. Then, 3.1\%, 1.1\%, 0.9\%, and 0.9\% protons are tagged as photon-like events at energies greater than $2,3,5$, and $10 \mathrm{EeV}$, respectively.

The period of the observed data set is from May 2008 to July 2013. In this work, I used the data obtained by two FD stations, BR and LR, and the SD array. Figure B 3 shows $X_{\max }$ of observed events together with the line denoting the photon-like event selection criterion. The events of which $X_{\max }$ 

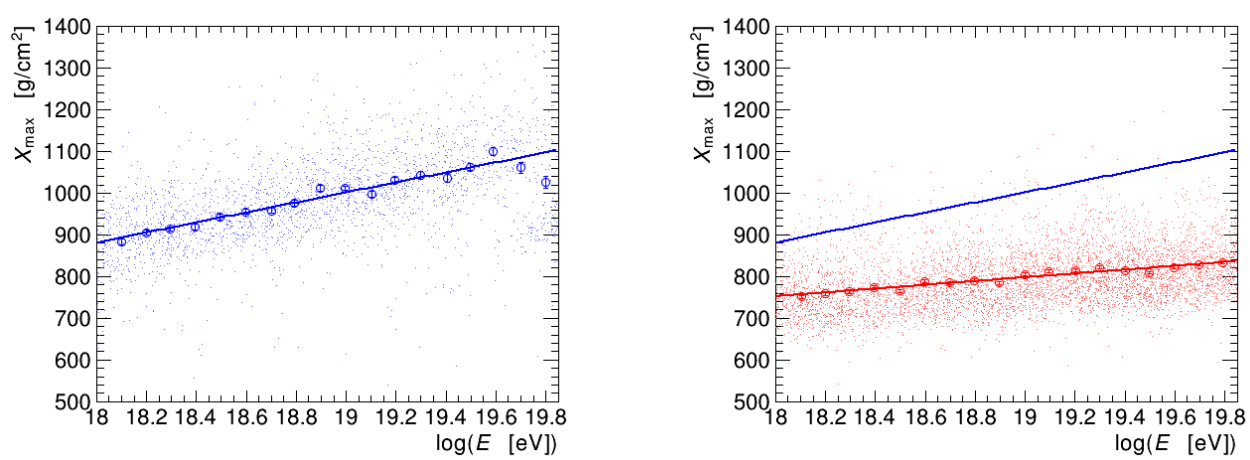

Figure 2: (Left) The blue dots are $X_{\max }$ values of photon-induced EASs. The blue open circles show the averaged $X_{\max }$ at each energy bin, the size of each bin is 0.1 in logarithm of energy. The blue line shows the fitting results of photon averaged $X_{\max }$. (Right) The red dots are reconstructed $X_{\max }$ for MC simulated events of primary protons, and blue line is the selection criterion determined by left figure. Open circles show the averaged $X_{\max }$ of protons at each energy bin, the size of each bin is 0.1 in logarithm of energy.

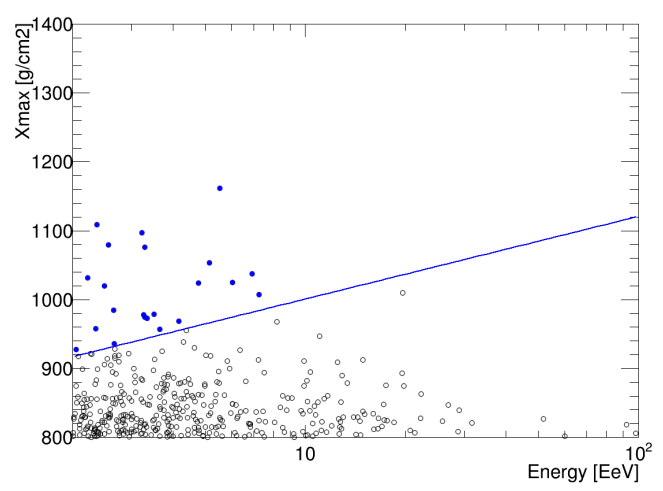

Figure 3: Reconstructed $X_{\max }$ of observed events and the selection criterion. The circles show data points and the line is the selection criterion described in this section. The observed data passed the quality cuts and the photon enhance cut.

values exceed the criterion line are selected as photon-like events. The numbers of the photon-like events are 22, 14, 5, and 0 in the energy ranges with energies greater than $2,3,5$, and $10 \mathrm{EeV}$, respectively. The numbers of all events which passed quality cuts are 1525, 830, 371, and 123 in the same corresponding energy ranges, respectively.

An individual photon-like event selected according to the criteria above can not be definitively identified as a photon. It is expected that some protons have deeper $X_{\max }$ than the selection criterion due to fluctuation of a longitudinal development. Thus, we compared the number of photon-like events with the expected number of photon-like event from deeply penetrating proton EASs. In order to estimate the proton contamination, the same selection is applied to the proton MC made with QGSJETII-03. As a result, the expected numbers of photon-like events with proton MC are $47.1,8.7,3.3$, and 1.1 with energy ranges grater than $2,3,5$, and $10 \mathrm{EeV}$, respectively. 


\section{Photon Fraction Upper Limit}

The upper limit of the photon fraction, which is the fraction of photon flux to all particle flux, is calculated with selection results in this section. In order to obtain a conservative upper limit, the photon-like events are assumed to be caused by primary photons. This is because the $X_{\max }$ distribution of protons highly depends on which high energy hadronic interaction model is used, thus we can not estimate realistic contamination from protons without systematic uncertainty.

The upper limit of the number of photon-like events using the Poisson distribution is calculated assuming no background, i.e., all the photon-like events are assumed to be photons. Poisson upper limits of the number of photon-like events with 95\% confidence level are obtained as 31.4, 21.9, 10.5 , and 3.0 in the range with energies above 2, 3, 5, and $10 \mathrm{EeV}$, respectively. As discussed above the photon selection of this analysis discards not only nuclei but also photons, thus the selection efficiency should be taken into account for calculating the upper limit. As a results we obtained 95\% confidence level upper limits of the numbers of photon-like events, $\left.n_{\gamma}\right|_{95 \%}$, as 66.0, 45.9, 21.0, and 6.3 in the range with energies above 2, 3, 5, and $10 \mathrm{EeV}$, respectively.

Finally, we obtained the upper limit of photon fraction with $95 \%$ confidence level, $\left.F_{\gamma}\right|_{95 \%}$, from the following relation,

$$
\left.F_{\gamma}\right|_{95 \%}=\frac{\left.I_{\gamma}\right|_{95 \%}}{\left.I_{\gamma}\right|_{95 \%}+I_{\mathrm{p}}}=\frac{\left.n_{\gamma}\right|_{95 \%}}{\left.n_{\gamma}\right|_{95 \%}+\left(n_{\mathrm{obs}}-n_{\gamma} \mid 95 \%\right) A_{\gamma} / A_{\mathrm{p}}},
$$

where $I_{\gamma}$ and $I_{\mathrm{p}}$ are the fluxes of photons and protons, respectively, $n_{\mathrm{obs}}$ indicates the number of observed events, $n_{\gamma}$ is the number of the photon-like events, $A_{\gamma}$ and $A_{\mathrm{p}}$ are the apertures of the experiment for primary photons and protons, respectively, and the notation of "95\%" means $95 \%$ confidence level upper limit. The ratio of the apertures, $A_{\gamma} / A_{\mathrm{p}}$, is substituted from the acceptance ratio derived in Section [5. Then, the upper limits of the photon fractiona with $95 \%$ confidence level are obtained as 12.7\%, 16.3\%,17.7\%, and 17.8\% in energies above 2, 3, 5, and $10 \mathrm{EeV}$, respectively. The results of the calculations are summarized on Table 1 . Figure $\mathbb{\text { G }}$ is the photon fraction upper limit comparing to other experimental results and predictions from several models. In this figure, the result from the same processes of analysis as this work with monocular data set, which are reconstructed with the monocular method, is also shown.

There are systematic uncertainties on energy determination with the hybrid analysis. It consists of uncertainties of the detector calibration, the atmospheric attenuation, the fluorescence yield, and the reconstruction error. The total systematic uncertainty on energy determination is calculated with the quadratic sum of these uncertainties, and that is $21 \%$. In order to estimate the systematic uncertainty on the photon fraction upper limits, the upper limits are recalculated with shifting the energy scales by $-42 \%,-21 \%,+21 \%$, and $+42 \%$. Then, we can find that systematic shift on upper limits in each energy range is $+2.5 \%,+0.0 \%,+1.8 \%$, and $+29.5 \%$ in energies above $2,3,5$, and $10 \mathrm{EeV}$.

\section{Conclusion and Future Work}

The studies for EASs of primary photons and searching for UHE photons using $X_{\max }$ with hybrid reconstruction technique are presented in this work. This is the first result which measured by the hybrid detection technique in the northern hemisphere. 
Table 1: The results of the photon fraction upper limits with $95 \%$ confidence level. See text for details.

\begin{tabular}{l|cccc}
\hline & $>2 \mathrm{EeV}$ & $>3 \mathrm{EeV}$ & $>5 \mathrm{EeV}$ & $>10 \mathrm{EeV}$ \\
\hline The number of photon-like events, $n_{\gamma}$ & 22 & 14 & 5 & 0 \\
Poisson fluctuation with 95\% c.l. & 31.4 & 21.9 & 10.5 & 3.0 \\
Photon selection efficiency & 0.476 & 0.477 & 0.500 & 0.479 \\
Upper limit of the number of photon- & 66.0 & 45.9 & 21.0 & 6.3 \\
like events with 95\% c.l. $n_{\gamma} \mid 95 \%$ & & & & \\
Acceptance ratio, $A_{\gamma} / A_{\mathrm{p}}$ & 0.312 & 0.302 & 0.280 & 0.248 \\
The number of all data, $n_{\text {obs }}$ & 1525 & 830 & 371 & 123 \\
The upper limit of the photon fraction & 12.7 & 16.3 & 17.7 & 17.8 \\
with 95\% c.l., $F_{\gamma} \mid 95 \%(\%)$ & & & & \\
\hline
\end{tabular}

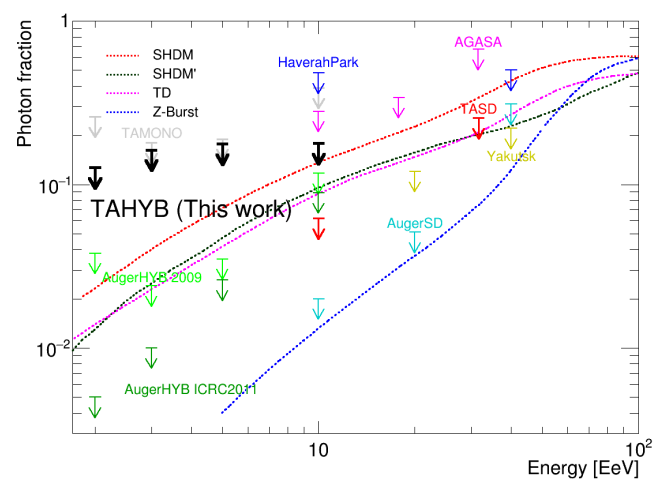

Figure 4: Photon fraction upper limits. Black arrows show the results of this work. The other arrows show other experimental results: gray is the TA monocular analysis result, red is the TA SD analysis result [[ए3], light green is the Auger hybrid result presented in 2009[एய], green is the Auger hybrid result presented in

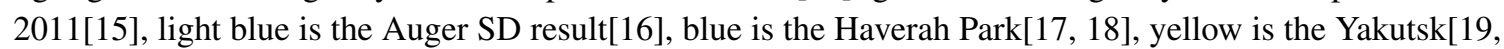
[20], and pink is the AGASA result[2], 22]. The lines show the predictions from top-down models [23], [24, [25].

As a result of this work, the predicted photon fractions with top-down scenarios are not constrained. However, in consideration of combination with the TA SD result, which search for photon candidates by using waveform feature collected by SDs, SHDM models and TD model of the UHECR generation are constrained with 95\% confidence level, and Z-burst model are survived with these upper limits in the northern hemisphere. It is important that independent search of UHE photons in the northern and the southern hemisphere, because UHE photons are expected to be anisotropically observed since sources or generation points of UHE photons are limited to near to the Earth due to their mean free path, and also they are not deflected by the magnetic fields in the universe.

In future analysis, we will include information of secondary particles at the ground, such as lateral distribution and shape of waveform derived as SD observable, and then it is expected that the discrimination power of photons from nuclei is improved, and a photon likeness of each event 
can be estimated.

\section{Acknowledgements}

The Telescope Array experiment is supported by the Japan Society for the Promotion of Science through Grantsin-Aid for Scientific Research on Specially Promoted Research (21000002) "Extreme Phenomena in the Universe Explored by Highest Energy Cosmic Rays" and for Scientific Research (19104006), and the Inter-University Research Program of the Institute for Cosmic Ray Research; by the U.S. National Science Foundation awards PHY-0307098, PHY0601915, PHY-0649681, PHY-0703893, PHY-0758342, PHY-0848320, PHY-1069280, PHY-1069286, PHY-1404495 and PHY-1404502; by the National Research Foundation of Korea (2007-0093860, R32-10130, 2012R1A1A2008381, 2013004883); by the Russian Academy of Sciences, RFBR grants 11-02-01528a and 13-02-01311a (INR), IISN project No. 4.4502.13, and Belgian Science Policy under IUAP VII/37 (ULB). The foundations of Dr. Ezekiel R. and Edna Wattis Dumke, Willard L. Eccles, and George S. and Dolores Doré Eccles all helped with generous donations. The State of Utah supported the project through its Economic Development Board, and the University of Utah through the Office of the Vice President for Research. The experimental site became available through the cooperation of the Utah School and Institutional Trust Lands Administration (SITLA), U.S. Bureau of Land Management, and the U.S. Air Force. We also wish to thank the people and the officials of Millard County, Utah for their steadfast and warm support. We gratefully acknowledge the contributions from the technical staffs of our home institutions. An allocation of computer time from the Center for High Performance Computing at the University of Utah is gratefully acknowledged.

This work is supported by the Japan Society for the Promotion of Science (JSPS) through Grant-in-Aid for JSPS Fellows.

\section{References}

[1] R. Abbasi et al., The Astrophysical Journal Letters, 790, L21, 2014.

[2] M. B. Hindmarsh and T. W. B. Kibble, Reports on Progress in Physics, 58, p. 477, 1984.

[3] M. Birkel and S. Sarkar, Astroparticle Physics, 9, pp. 297-309, 1998.

[4] T. J. Weiler, Astroparticle Physics, 11, pp. 303-316, 1999.

[5] T. Abu-Zayyad et al., Nuclear Instruments and Methods in Physics Research A, 676, pp. 54-65, 2012.

[6] T. Abu-Zayyad et al., Nuclear Instruments and Methods in Physics Research A, 689, pp. 87-97, 2012.

[7] D. Heck et al., CORSIKA: A Monte Carlo Code to Simulate Extensive Air Showers, Forschungszentrum Karlsruhe Report FZKA 6019, 1998.

[8] S. Ostapchenko, Nuclear Physics B Proceedings Supplements, 151, pp. 143-146, 2006.

[9] B. T. Stokes et al., Astroparticle Physics, 35, pp. 759-766, 2012.

[10] T. Erber, Review of Modern Physics, 38, pp. 626-659, 1966.

[11] T. Abu-Zayyad et al., Astroparticle Physics, 61, pp. 93-101, 2015.

[12] T. Fujii et al., AIP Conference Proceedings, 1367, pp. 149-152, 2011.

[13] T. Abu-Zayyad et al., Physical Review D, 88, 112005, 2013.

[14] The Pierre Auger Collaboration, Astroparticle Physics, 31, pp. 399-406, 2008.

[15] K. Kampert et al., "Highlights from the Pierre Auger Observatory", Proceedings of 32st International Cosmic Ray Conference, 2011.

[16] J. Abraham et al., Astroparticle Physics, 29, pp. 243-256, 2008.

[17] M. Ave et al., Physical Review Letters, 88, pp. 2244-2247, 2000.

[18] M. Ave et al., Physical Review D, 65, 063007, 2002.

[19] A. V. Glushkov et al., JETP Letters, 85, pp. 131-135, 2007.

[20] A. V. Glushkov et al., Physical Review D, 82, 041101, 2010.

[21] K. Shinozaki et al., The Astrophysical Journal, 571, L117, 2002.

[22] M. Risse et al., Physical Review Letters, 95, 171102, 2005.

[23] G. Gelmini et al., Journal of Experimental and Theoretical Physics, 106, 11, 2008.

[24] J. Ellis et al., Physical Review D, 74, 115003, 2006.

[25] M. Risse et al., Physics Letters A, 22, 749, 2007. 